\title{
Size and distance judgments under reduced conditions of viewing'
}

\author{
WILLIAM EPSTEIN, ${ }^{2}$ UNIVERSITY OF WISCONSIN \\ ALI A. LANDAUER, ${ }^{3}$ UNIVERSITY OF WESTERN AUSTRALIA
}

\begin{abstract}
Magnitude estimations of the size and distance of a variable relative to a standard were obtained in the absence of distance cues. Estimates were provided by different groups under three conditions: (a) physical size and distance variant, visual angle of the variable constant and equal to the standard, (b) physical size constant, physical distance and visual angle of variable changing, and (c) physical distance constant, physical size and visual angle of the variable changing. The results in both experiments were very similar. In each case both size and distance estimates conformed to relative visual angle. The results are applied to an analysis of size-matches that are obtained when distance cues are eliminated.
\end{abstract}

In the absence of distance cues, visual angle size matches are obtained (Holway \& Boring, 1941; Baird, in press). This fact has been construed in terms of the size distance invariance hypothesis: In the absence of distance cues, there is a tendency for the standard and variable to appear equidistant and as a result the standard and variable will appear the same size when they subtend equal visual angles (Epstein \& Park, 1961; Wallach \& McKenna, 1960). An evaluation of the invariance hypothesis calls for data concerning distance perception under reduced conditions of viewing. Künnapas's (1968) experiment was designed to provide magnitude estimations of the distance of a varible relative to a fixed standard. His Condition R1 eliminated all distance cues, with the exception of accommodation. A standard presented at a constant distance was assigned the number 10 , and $S$ estimated the distance of a variable presented at each of seven distances. At each distance the visual angle of the variable was constant and equal to the visual angle of the standard. Under Condition R2 the physical size of the variable was constant so that variations of distance were accompanied by variations of relative visual angle. Under Condition $R 1$ Kunnapas found that the distance estimates did not vary with physical distance. At each distance the mean estimate was approximately 10 , the value assigned to the standard. Under Condition R2 distance estimates tended to be accurate.

Künnapas's findings for Condition R1 seem to confirm the invariance hypothesis as an account of the visual-angle size matches obtained under reduced conditions. But there is another plausible interpretation. In this account, visual-angle size matches under reduced conditions are attributed to direct unmediated determination of perceived size by visual angle (e.g., Rock \& McDermott, 1964): According to this view, perceived size under Condition R1 is determined directly by retinal size without the interaction of perceived distance. To explain the distance judgments reported by Kunnapas we need only assume that judged distance is determined independently by relative visual angle. Obviously, relative visual angle determines relative perceived distance under Condition R2. Condition R1 simply represents the limiting case of zero difference between the visual angles subtended by the standard and the variable and consequently equidistance judgments are obtained.

At issue is the conclusion we are justified to draw from Künnapas's results. Do the findings establish the claim that visual-angle size matches depend on perceived equidistance, or is the alternative account still tenable? It is not possible to determine which interpretation is correct if we consider only Condition R1, since both interpretations have identical expectations for size and distance estimates under this condition. But Condition R2 does offer the opportunity to decide between them. The size-distance invariance hypothesis predicts that if $S$ is asked to judge both size and distance under Condition R2, he will judge one of the two properties to vary in proportion to relative visual angle, and he will judge the other property to be constant. For example, if distance estimates vary with variations of relative visual angle, then size estimates should remain constant. This outcome would conform to the model of size-distance interaction that is presumed to underlie the visual-angle size matches under Condition R1. The alternative interpretation predicts that under Condition $\mathrm{R} 2$, both relative distance and relative size estimates will conform to relative visual angle, e.g., the standard will be estimated to be nearer and larger or farther and smaller. This covariation has been called the "size-distance paradox" (Epstein $\&$ Park, 1961) and is inconsistent with the invariance hypothesis.

Two experiments were designed to explore this question. In each experiment magnitude estimations of both size and distance were obtained under three conditions: Condition 1-Distance of the variable was varied, but visual angle was constant and equal to the standard. Condition 2-Distance of the variable was varied, but physical size was constant, so that variations of relative visual angle were produced. Condition 3-The standard and the variable were equidistant at a constant distance, but the physical size of the variable was varied to produce relative visual angle relationships comparable to those present under Condition 2. Condition 3 was introduced as a check on the experimental arrangement and the analysis of Condition 2 . If all distance cues are indeed absent and relative visual angle determines the size and distance estimates, then the results for Condition 3 should be comparable in all respects to those obtained under Condition 2.

\section{EXPERIMENT 1}

\section{Apparatus}

In each of the three conditions a standard was presented at a distance of $115 \mathrm{~cm}, 17.5 \mathrm{deg}$ to the right of S's median plane. The standard was an electroluminescent disk, $24 \mathrm{~mm}$ in diam, that subtended a visual angle of $1.20 \mathrm{deg}$. At the same angular separation to the left of $S$ the variable stimuli were presented. These were always electroluminescent disks shown in exactly the same line of sight and at the same height, $35 \mathrm{deg}$ to the left of the standard. Phosphorescent colors (self-luminous disks) like those used by Künnapas are not entirely suitable since a distant disk with a smaller visual angle appears dimmer than a nearer one, thus giving $S$ an important distance cue. This is a potential source of confounding in Künnapas's study.

Condition 1 duplicated Künnapas's (1968) experimental arrangement $R 1$. The $S$ viewed seven different variable disks that had diameters of $5,9,16,24,41,62$, and $82 \mathrm{~mm}$ and that were presented individually at viewing distances of $25,45,75,115$, 195,295 , and $395 \mathrm{~cm}$, respectively. In each of these 
Table 1

Mean Size and Mean Distance Estimates

\begin{tabular}{|c|c|c|c|c|c|c|c|}
\hline \multirow[b]{2}{*}{ Condition } & \multirow{2}{*}{$\begin{array}{c}\text { Size of } \\
\text { Variable } \\
\text { (mm) }\end{array}$} & \multirow{2}{*}{$\begin{array}{c}\text { Distance of } \\
\text { Variable } \\
\text { (cm) }\end{array}$} & \multirow{2}{*}{$\begin{array}{l}\text { Visual } \\
\text { Angle } \\
\text { (deg) }\end{array}$} & \multicolumn{2}{|c|}{ Experiment 1 } & \multicolumn{2}{|c|}{ Experiment 2} \\
\hline & & & & $\begin{array}{c}\text { Mean Size } \\
\text { Estimate }\end{array}$ & $\begin{array}{c}\text { Mean Distance } \\
\text { Estimate }\end{array}$ & $\begin{array}{c}\text { Mean Size } \\
\text { Estimate }\end{array}$ & $\begin{array}{c}\text { Mean Distance } \\
\text { Estimate }\end{array}$ \\
\hline 1 & $\begin{array}{r}5 \\
9 \\
16 \\
24 \\
41 \\
62 \\
82\end{array}$ & $\begin{array}{r}25 \\
45 \\
75 \\
115 \\
195 \\
295 \\
395\end{array}$ & $\begin{array}{l}1.20 \\
1.20 \\
1.20 \\
1.20 \\
1.20 \\
1.20 \\
1.20\end{array}$ & $\begin{array}{l}9.63 \\
7.92 \\
8.46 \\
9.58 \\
9.38 \\
8.21 \\
8.83\end{array}$ & $\begin{array}{l}8.79 \\
9.50 \\
8.83 \\
9.71 \\
8.58 \\
9.79 \\
9.29\end{array}$ & $\begin{array}{l}9.63 \\
9.25 \\
9.38 \\
9.79 \\
9.60 \\
9.43 \\
9.35\end{array}$ & $\begin{array}{r}7.94 \\
9.54 \\
9.75 \\
9.81 \\
9.84 \\
10.44 \\
10.53\end{array}$ \\
\hline 3 & $\begin{array}{r}82 \\
62 \\
41 \\
24 \\
16 \\
9 \\
5 \\
\end{array}$ & $\begin{array}{l}115 \\
115 \\
115 \\
115 \\
115 \\
115 \\
115\end{array}$ & $\begin{array}{l}4.09 \\
3.09 \\
2.04 \\
1.20 \\
0.80 \\
0.45 \\
0.25 \\
\end{array}$ & $\begin{array}{r}20.75 \\
15.12 \\
12.04 \\
9.33 \\
6.91 \\
3.37 \\
2.04\end{array}$ & $\begin{array}{r}4.33 \\
4.87 \\
7.29 \\
9.83 \\
11.87 \\
16.50 \\
19.91\end{array}$ & $\begin{array}{r}19.99 \\
16.24 \\
12.41 \\
9.64 \\
6.81 \\
3.80 \\
2.41\end{array}$ & $\begin{array}{r}5.18 \\
5.99 \\
7.56 \\
10.11 \\
11.84 \\
14.01 \\
16.74\end{array}$ \\
\hline
\end{tabular}

combinations of stimulus size and viewing distance the variable subtended a constant visual angle of $1.20 \mathrm{deg}$.

In Conditions 1 and 2 the disks were made by covering an electroluminescent panel with a sheet of thin metal that had a central circular aperture of the desired diameter. The panels were attached to boards that could be lowered into S's field of vision and activated by remote control. In its essential details the apparatus and method described by Künnapas was adopted. The amount of luminous flux inpinging on S's eye was so low that it could not be measured with available light meters.

Condition 2 duplicated Künnapas's experimental arrangement R2. All seven variable disks had a diameter of $24 \mathrm{~mm}$ and subtended visual angles of $5.50,3.06,1.83,1.20,0.71,0.47$, and $0.35 \mathrm{deg}$ when presented at the same viewing distances as in Condition 1. From S's position the brightness of all stimuli was subjectively equal. Phenomenal brightness equality was obtained by taking the mean subjective brightness match of the two Os and adjusting the voltage of each electroluminescent panel by a separate variable resistance. Under the low illumination condition of the experiment, this was the most practical procedure for equating brightness.

In Condition 3 seven disks of different size were presented individually at the same distance as the standard $(115 \mathrm{~cm})$. Chain drives and a shaft interacted with a large rotating circular metal shield which had round apertures of $5,9,16,41,62$, and $82 \mathrm{~mm}$ diam at various positions. By remote control $E$ could adjust the shield so that one of its apertures was precisely positioned in front of an electroluminescent panel. The display visible to $S$ was a luminous disk subtending a visual angle of $4.09,3.09,2.04$, $1.20,0.80,0.45$, or $0.25 \mathrm{deg}$ and appearing in the same line of sight at which the variable was visible in the other experimental conditions. As far as retinal stimulation is concerned, Condition 3 was analogous to Condition 2 since disks subtending different visual angles could be presented to $S$.

\section{Subjects}

Three groups of $12 \mathrm{Ss}$ were used, one for each experimental condition. All Ss were students from an introductory course in psychology who served as unpaid $S$ s and who volunteered in order to fulfill an optional course requirement. All Ss had uncorrected binocular vision; their ages ranged from 18 to 26 years (mean 19.4).

\section{Procedure}

The Ss were tested individually and were assigned to an experimental condition in order of their arrival. The $S$ was met in a waiting room located in a different wing and on a different floor from the laboratory. The S's left eye was occluded by an eye patch and a blindfold placed over both eyes. He was then led to the laboratory where the blindfold was removed in total darkness. The $\mathbf{S}$ was seated and his head was positioned in a combined chin and head rest that prevented gross head movements. In addition, $S$ was told not to move his head during and between trials. A viewing aperture on the headrest permitted observation through S's right eye only. The procedure of blindfolding $S$ in another room was adopted to prevent $S$ from



Fig. 1. Mean size and mean distance estimates made under Conditions 2 and 3 in Experiment 1, plotted against a logarithmic transformation of the visual angle subtending at $S$ 's eye. (Each point is based on four estimates made by 12 different Ss.) 


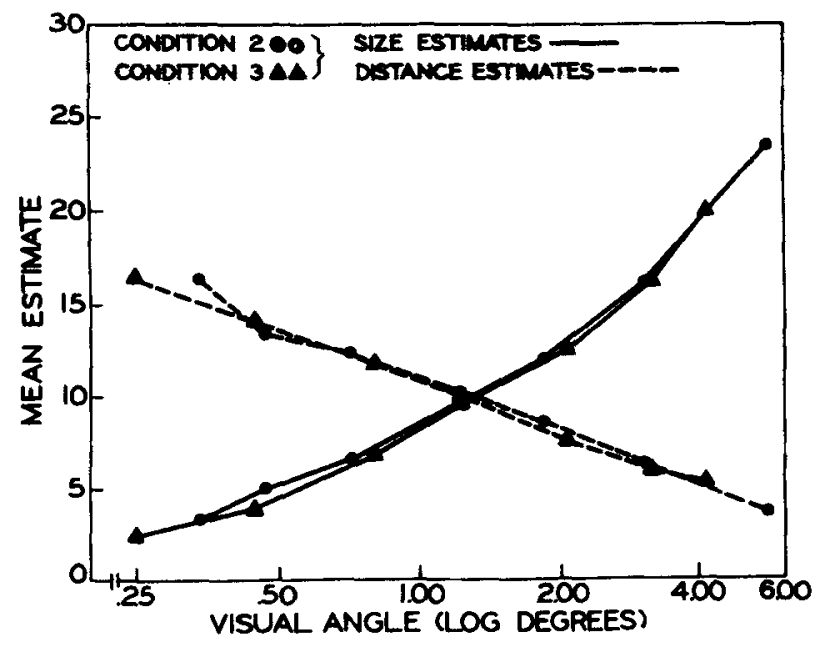

Fig. 2. Mean size and mean distance estimates made under Conditions 2 and 3 in Experiment 2, plotted against a logarithmic transformation of the visual angle subtending at S's eye. (Each point is based on four estimates made by 20 different Ss.)

obtaining information about the size of the laboratory. It also helped to reduce the time for dark adaptation, which was not systematically controlled, but which lasted for at least $8 \mathrm{~min}$ in total darkness before the first observation was made. At the conclusion of the experiment, $S$ was again blindfolded in darkness before being led back to the waiting room.

The method of magnitude estimation was used. The size and distance of the standard were assigned a value of 10 and $S$ was instructed to estimate the size or distance of the variable by assigning an appropriate number. Each $S$ provided 28 estimates, 14 for distance and 14 for size. Half of the Ss supplied all 14 size estimates before the distance estimates were solicited; the remaining Ss gave the distance estimates first. The order of stimulus presentation was arranged in four randomized blocks of seven trials. No audible sounds accompanied changing of the stimuli. The standard and variable disks were activated only when the variable was in position. Both were extinguished after each estimate.

\section{Results}

Table 1 shows the means of the untransformed estimates made under the three conditions, together with the dimensions of the variable. Figure 1 presents the mean size and distance estimates under Conditions 2 and 3 plotted against a logarithmic transformation of the visual angle subtending at $S$ 's eye. The logarithmic transformation was used to emphasize differences in smaller visual angles.

The distance estimates under Conditions 1 and 2 are similar to those reported by Künnapas. Under Condition 1 the mean estimates ranged from 8.58 to 9.97 , while the actual distances varied from 25 to $395 \mathrm{~cm}$. The mean estimates are close to the value of 10 assigned to the standard. Under Condition 2 the mean distances varied systematically with variations of visual angle; the estimates ranged from 2.63 to 23.96 . The same trend was observed under Condition 3. As Fig. 1 shows, the functions relating distance estimation to visual angle are comparable under Conditions 2 and 3 . We may conclude that distance estimates are predictable from visual angle. The distance estimates under
Condition 1 can be similarly interpreted. Physical size and physical distance are not good predictors.

Table 1 shows that the size estimates under Condition 1 ranged from 7.92 to 9.63 while the actual sizes varied from 5 to $82 \mathrm{~mm}$. Figure 1 shows that the size estimates under Conditions 2 and 3 were very similar. In both cases estimated size varied directly with visual angle.

\section{EXPERIMENT 2}

Experiment 2 differed from Experiment 1 in only one respect. In Experiment 1 the instructions first solicited estimates of one dimension, e.g., size, and only after all of these estimates were completed, was S's attention called to the other dimension, e.g., distance. It is possible that the results for Conditions 2 and 3 are due to the fact that the instructions focused S's attention separately on size and distance and that the procedure introduced a relatively long temporal separation between the size and distance estimates of each stimulus. In Experiment 2 the instructions and order of estimations was modified to check this possibility.

\section{Subjects}

Sixty Ss were drawn from the same population that supplied Ss for Experiment 1. Twenty Ss served under each of the three experimental conditions.

\section{Procedure}

Instructions were administered to encourage $S$ to attend to size and distance simultaneously. The essential portion of the instructions was as follows:

"Estimate the size and the distance of the disk on your left, as compared with the distance and size of the disk on your right, to whose size as well as distance away from you, I want you to assign the arbitrary value of 10 . Thus, if the disk on your left appears smaller than the one on your right, you should estimate its size with a number which is less than 10 . At the same time you should also estimate the distance of the disk on your left and compare it with the distance of the disk on your right. If the left disk seems closer than the one on your right, you should estimate its distance with a number which is less than 10 ; if both disks look equidistant your distance estimate should be 10 ; and if the disk on your left appears to be further away, your distance estimate should be a number which is greater than 10. Always estimate both the size and the distance at the same time, and when giving your estimates, give your distance estimate first and clearly indicate which numerical value refers to distance and which to size."

Each S provided 28 estimates of size and distance. Half of the Ss were instructed to give their size estimates first on the initial 14 trials and their distance estimates first on the second 14 trials. For the other Ss the order was reversed. The order of stimulus presentation was arranged in four randomized blocks of seven trials.

\section{Results}

The data were examined to determine whether or not any differences were associated with the various orders of reporting. No differences were detected. Therefore, all the estimates for a given stimulus and condition were combined. The mean estimates of size and distance under the three experimental conditions for each of the seven different stimulus configurations are shown in Table 1. Figure 2 shows the mean estimates given in Conditions 2 and 3 plotted against a logarithmic transformation of the visual angle. 
The data show that the mean estimates under all conditions were dependent upon the visual angle subtended by the variable. The small irregularities observed when the stimuli were presented at a short distance from the eye are probably due to the operation of accommodation cues. A variable subtending a smaller visual angle than the standard was estimated to be both smaller and further away, while a variable that subtended a larger visual angle than the standard was estimated to be both larger and closer. When the variable and standard subtended the same visual angle, the two displays tended to be estimated as equidistant and of similar size. All these estimates were independent of the veridical nature of the display: of the physical size and distance of the variable.

All the findings of Experiment 1 were confirmed in Experiment 2. A number of irregularities present in the data of Experiment 1 (Fig. 1) have been eliminated by the procedural innovations of Experiment 2.

\section{DISCUSSION}

The results for distance estimation under Condition 1 confirmed Kunnapas's (1968) findings. This was true both for Experiments 1 and 2 despite the difference between the estimation procedures used by Künnapas and the procedure in Experiment 2. The results for size estimation under Condition 1 conformed to the findings that have been reported in the size-perception literature. Visual angle matches were made at all distances of the variable. These findings are perfectly compatible with the invariance hypothesis.

The results for distance estimation under Condition 2 are also in good agreement with Künnapas's findings. The fact that highly comparable results were obtained under Condition 3 is further evidence that the distance estimates are based on relative visual angle. The new finding was that the size estimates also covaried in a manner predictable from relative visual angle. Variables that subtended larger visual angles than the standard were estimated as nearer and larger; variables that subtended smaller visual angles than the standard were estimated as more distant and smaller. This outcome was obtained in both experiments under both Conditions 2 and 3 . These results are inconsistent with the invariance interpretation of the data obtained under Condition 1. To be consistent with this interpretation, either distance estimates or size estimates should have been constant under Conditions 2 and 3 . If distance estimates varied in agreement with changes of visual angle, then size estimates should have been invariant, or vice versa. None of the Ss conformed to this requirement.

If the invariance hypothesis is ruled out as an account of our data, what altemative is plausible? The simplest account of our data, as well as the results of Künnapas's (1968) Condition R1, is that both size estimates and distance estimates are directly determined by relative visual angle. Inasmuch as, in the absence of other distance cues, a difference in visual angle is compatible with a judged difference in distance or size, $S$ avails himself of both alternatives depending on the task requirement. If the $\mathrm{E}$ solicits a size judgment, $S$ translates the visual-angle difference into a size judgment, proportional to visual angle. If a distance judgment is solicited, $\mathbf{S}$ translates the visual-angle difference into a distance judgment, proportional to visual angle. But in neither case does one judgment depend on the other judgment. The size and distance judgments are independent products of two ways of processing relative visual angle, and the judgments that were obtained can be accounted for solely on the basis of visual-angle considerations. In this sense, the results are more compatible with the hypothesis that visual angle can directly determine perceived size (Rock \& McDermott, 1964).

\section{REFERENCFS}

BAIRD, J. C. Psychophysical analysis of visual space. London: Pergamon Press, in press.

EPSTEIN, W., \& PARK, J. The current status of the size-distance hypotheses. Psychological Bulletin, 1961, 58, 491-514.

HOLWAY, A. H., \& BORING, E. G. Determinants of apparent visual size with distance variant. American Journal of Psychology, 1941, 51, 21-37.

KÜNNAPAS, T. Distance perception as a function of available distance cues. Journal of Experimental Psychology, 1968, 77, 523-529.

ROCK, I., \& MCDERMOTT, W. The perception of visual angle. Acta Psychologica, 1964, 22, 119-134.

WALLACH, H., \& MCKENNA, V. V. On size-perception in the absence of distance cues. American Journal of Psychology, 1960, 73, 458-460.

\section{NOTES}

1. This research was supported by Public Health Service Research Grant No. 16390-01 from the National Institute of Mental Health to the first author.

2. Address: Department of Psychology, The University of Wisconsin, Madison, Wisconsin 53706.

3. The research was conducted while A.A.L. was on leave from the University of Western Australia. He is indebted to the Department of Psychology of the University of Wisconsin for its cooperation in all aspects of the research.

(Accepted for early publication May 28, 1969.) 